\title{
VOXEL-BASED MORPHOMETRY ANALYSIS OF GRAY MATTER ON QURAN MEMORIZERS
}

Iqbal Jamaludin ${ }^{1}$, Mohd Zulfaezal Che Azemin ${ }^{1}$, Abdul Halim Sapuan ${ }^{1}$ and Radhiana Hassan $^{1}$

${ }^{1}$ International Islamic University Malaysia, Pahang, Malaysia.

Presenter: Iqbal Jamaludin, iqbalbinjamaludin@gmail.com

Introduction: The brain is the most complex organ in the human body. Robust and vigorous daily activities may cause changes to the brain structure. Huffaz, individuals who memorise the Quran undergo intensive memorization training which may lead to structural changes in specific regions of the brain.

Materials and method: This study looked at possible change that occurred on gray matter by characterising the textual memorization of brain structure using voxel-based morphometry (VBM). It involves voxel-by-voxel comparison of gray matter intensity of the MRI images. Forty-seven subjects ( 23 huffaz, 24 non-huffaz) aged between 21-25 years were voluntarily recruited. Subjects were scanned by 3 Tesla MRI system. Images were then re-aligned according to standardised Montreal Neurological Institute (MNI) coordinates. The MRIs were then segmented into gray matter, white matter and cerebrospinal fluid. Independent sample t-test was performed between the two groups.

Results: No significant difference was found between the brain region of the huffaz and non-huffaz with appropriate corrections for family-wise error (FWE) at a threshold of $p$ $=0.05$. However, with a more lenient criteria $(p=0.001$, uncorrected, cluster size $=50$ $\mathrm{mm}^{3}$ ), we found that gray matter volume in Brodmann Area 6 and Brodmann Area 7 of the huffaz were significantly higher than the non-huffaz group.

Conclusion: VBM is not sensitive enough to detect complex anatomical differences between huffaz and non-huffaz with the current sample size. Future study to explore possible image processing tools that can measure subtle structural change in human brain is warranted. 